\title{
The miRNA let-7a1 inhibits the expression of insulin-like growth factor 1 receptor (IGF1R) in prostate cancer PC-3 cells
}

\author{
Li-Na Wang*, Wei-Wen Chen*, Ju Zhang, Chao-Yang Li, Chun-Yan Liu, Jing Xue, Peng-Ju Zhang and An-Li Jiang
}

Reduced microRNA (miRNA) let-7a expression and the activation of insulin-like growth factor-1 receptor (IGF1R) signalling are both involved in prostate cancer and progression. In the present study, we demonstrated that the growth inhibitory effect of let-7a1 is directly related to targeting IGF1R gene expression in PC-3 cells. TargetScan predicted three potential target sites (T1, T2 and T3) of let-7a in the 3' untranslational region (3' UTR) of IGF1R mRNA. Real-time PCR, Western blot and luciferase reporter assays were used to detect the effects of let-7a1 overexpression or let-7a1 inhibitor on the IGF1R gene expression in PC-3 cells. The results indicated that let-7a1 could inhibit IGF1R expression by directly targeting the T1 and T2 sites in the 3' UTR of the IGF1R mRNA. We then used RT-PCR, luciferase reporter assays, 3-(4,5-dimethylthiazol-2-yl)-2,5- diphenyl-2 $H$-tetrazolium bromide (MTT) assay, flow cytometry and Hoechst 33342 staining to examine whether let-7a1-mediated inhibition of IGF1R expression also affects the IGF1R-mediated signalling events, including Elk1 activity and $c$-fos gene expression, proliferation, apoptosis and cell cycle. We demonstrated that let7a1-mediated IGF1R downregulation was accompanied by attenuation of Elk1 activity and $c$-fos expression, inhibition of cell proliferation, enhanced apoptosis and cell cycle arrest, and that loss function of let-7a1 via inhibition can upregulate IGF1R accompanied by an increase of Elk1 activity and $c$-fos expression, thereby enhancing cell proliferation. Altogether, these findings suggest that let-7a may be novel therapeutic candidate for prostate cancer.

Asian Journal of Andrology (2013) 15, 753-758; doi:10.1038/aja.2013.84; published online 26 August 2013

Keywords: IGF1R; microRNA (miRNA); prostate cancer

\section{INTRODUCTION}

Prostate cancer is one of the most common cancers ${ }^{1}$ and responds well to androgen ablation initially, before invariably progressing to treatment resistance characterized by a high proliferation rate and strong metastasis propensity. ${ }^{2,3}$ The progression of these tumours is reportedly facilitated by growth factors activating critical signalling cascades, thereby promoting prostate cancer cell growth, survival and migration. ${ }^{4}$ Among these growth and survival signalling pathways, the insulin-like growth factor-1/its receptor (IGF-1/IGF1R) system has been suggested to play a key role in the malignant transformation of prostate cancer cells. $^{5-8}$ IGF-1 binding to IGF1R results in tyrosine kinase activation and the stimulation of downstream signalling pathways that regulate proliferation, cell cycle and apoptosis. Several studies have reported that IGF1R expression is elevated in metastatic prostate cancer and hormone resistance progression. ${ }^{9-11}$ Therefore, inhibition of IGF1R signalling may provide an alternative approach to prostate cancer treatment.

MicroRNAs (miRNAs) are $\sim 22$ nucleotides long, non-coding and endogenous RNA molecules with important functions in development, cell differentiation, regulation of cell cycle and apoptosis. miRNAs exert their functions by base pairing with target mRNAs to regulate protein-coding gene expression via mRNA degradation or translation inhibition. More than $60 \%$ of human protein coding genes have been under selective pressure to maintain pairing to miRNAs, which suggests that most mammalian mRNAs are conserved targets of miRNAs. ${ }^{12}$ Much evidence demonstrates that miRNAs play important roles in the establishment and progression of human tumours. The miRNAs act as either oncogenes ${ }^{13-15}$ or tumour suppressors. ${ }^{16-18}$ The miRNA let-7a has been characterised as a tumour suppressor in several human cancers. ${ }^{19-24}$ Dong et al. ${ }^{25}$ reported that let-7a is downregulated in resected prostate cancer samples and in prostate cancer cells. PC-3 and DU145 cells (androgen-independent) express less let-7a than LNCaP cells (androgen-dependent). Target prediction algorithms indicate that let-7a1 can target a number of oncogenes, including IGF1R. Several studies have reported that the activation of IGF1R signalling is critical for prostate cancer cell growth and progression. In this study, we examine whether let-7a1 targets IGF1R expression to inhibit the proliferation of prostate cancer cells.

\section{MATERIALS AND METHODS}

Plasmid construction

Three miRNA let-7al targeting sequences were identified in the $3^{\prime}$ untranslational region ( $3^{\prime}$ UTR) of IGF1R mRNA. They are located at position 99-105, 2619-2626, 6661-6667 of the IGF1R 3' UTR. The targeting sequences and their mutants (shown in the following list) 
were synthesised and cloned into a pMIR-Report vector (Ambion, Grand Island, NY, USA) to form pMIR-T1, pMIR-T2 pMIR-T3, pMIR-T1m, pMIR-T2m and pMIR-T3m.

IGF1R-3' UTR-T1F: 5'-cCCATTCACAAGCCTCCTGTACCTCAGTGa-3'

IGF1R-3' UTR-T1R: 5' -agcttCACTGAGGTACAGGAGGCTTGTGAATGGgagct-3'

IGF1R-3' UTR-T2F: 5'-cTTCCCCCAAACATTTATCTACCTCACTCa-3'

IGF1R-3' UTR-T2R: $5^{\prime}$-agcttGAGTGAGGTAGATAAATGTTTGGGGGAAgagct-3'

IGF1R-3' UTR-T3F: 5' -cAGGTTTGCCAGAGTTTGTCTACCTCTGGa-3'

IGF1R-3' UTR-T3R: 5' -agcttCCAGAGGTAGACAAACTCTGGCAAACCTgagct- $3^{\prime}$

IGF1R-3' UTR-T1mF: 5'-cCCATTCACAAGCCTCCTGATGGAGTGTGa-3'

IGF1R-3' UTR-T1mR:5' -agcttCACTCTCCATCAGGAGGCTTGTGAATGGgagct-3'

IGF1R-3' UTR-T2mF: 5' -cTTCCCCCAAACATTTATGATGGAGACTCa-3'

IGF1R-3' UTR-T2mR: 5' -agcttGAGTCTCCATCATAAATGTTTGGGGGAAgagct-3'

IGF1R-3' UTR-T3mF: 5'-cAGGTTTGCCAGAGTTTGTGATGGAGTGGa-3'

IGF1R-3' UTR-T3mR: 5' -agcttCCACTCCATCACAAACTCTGGCAAACCTgagct- $3^{\prime}$

For construction of the miRNA let-7a1 expression plasmid, the let$7 a 1$ precursor was obtained by RT-PCR amplification using primers 7a1F (5' -cccggatccCCTGGATGTTCTCTTCACTG-3') and 7a1R (5' cccaagcttGCCTGGATGCAGACTTTTCT- $3^{\prime}$ ) and cloned into a pSilencer 4.1-CMV neo vector (Ambion) to form pSilencer4.1-let7a1.

For construction of the Elk1-reporter plasmid, the Elk1 element DNA sequences (Elk1-S: cTTCCCCCAAACATTTATCTACCTCACTCa, Elk1AS: agcttGAGTGAGGTAGATAAATGTTTGGGGGAAgagct) was synthesised and inserted upstream of the TATA box of the pGL4-LUC2[minp] vector (Premega, Madison, WI, USA) to form pGL4-Elk1.

\section{Cell culture and transfection}

The prostate cancer cell line PC-3 cells were maintained in RPMI-1640 medium (Gibco, Carlsbad, CA, USA), which was supplemented with $10 \%$ foetal bovine serum, 100 units $\mathrm{ml}^{-1}$ penicillin and $100 \mathrm{mg} \mathrm{ml}^{-1}$ streptomycin at $37{ }^{\circ} \mathrm{C}$ with $5 \% \mathrm{CO}_{2}$. Cell transfections were performed using FuGENE ${ }^{\circledR}$ HD (Roche, Mannheim, Germany) or siPORT NeoFX Transfection Agent (Ambion) according to the manufacturer's instructions. For RT-PCR and Western blot analysis, the cells were grown in 6well plates and transfected for $48 \mathrm{~h}$ with either pSilencer4.1-let7al or parental vector, with either let-7al inhibitor or normal control (NC) inhibitor (Ambion). For the luciferase reporter assay, the cells were grown in 24-well plates to $80 \%$ confluence and cotransfected for $48 \mathrm{~h}$ with pSilencer4.1-let7a1 (or parental vector) and pMIR-T (pMIR-T1, pMIR-T2, pMIR-T3, pMIR-T1m, pMIR-T2m, pMIR-T3m) plasmids (or parental vector) and pRL-TK Renilla luciferase vector (Promega) as an internal control. For the proliferation and apoptosis assays, the cells were grown in 96-well plates and transfected with either pSilencer4.1let7a1 or parental vector, with either let-7al inhibitor or NC inhibitor.

\section{Western blot analysis}

Western blotting was performed to detect IGF1R protein expression. Whole-cell lysates were prepared using lysis buffer (containing
$50 \mathrm{mmol}^{-1}$ Tris-Cl, pH 8.0, $150 \mathrm{mmol} \mathrm{l}^{-1} \mathrm{NaCl}, 0.1 \%$ SDS, $1 \%$ NP-40 and $100 \mu \mathrm{g} \mathrm{ml}^{-1}$ PMSF). Protein concentration was determined using the Bradford assay (Bio-Rad, Hercules, CA, USA). Extracted protein $(50 \mu \mathrm{g})$ was processed in a $10 \%$ SDS-PAGE and electrophoretic transferred to a polyvinylidene fluoride membrane. IGFIR protein expression was determined using an IGF1R $\beta$-subunit polyclonal antibody (Santa Cruz Biotechnology, Dallas, TX, USA). $\beta$-Actin (Sigma, St Louis, MO, USA) was used as an internal control. Immunoblots were detected using an ECL kit (Santa Cruz Biotechnology) and were visualised after exposure to $\mathrm{X}$-ray film.

\section{RNA extraction and real-time RT-PCR analysis}

Total RNA was extracted from PC-3 cells using Trizol reagent (Invitrogen, Grand Island, NY, USA) according to the manufacturer's instructions. First strand cDNA was synthesized using a RevertAid First Strand cDNA Synthesis Kit (Fermentas, Pittsburgh, PA, USA). The real-time PCR primers for amplification of miRNA let-7a1 used were $7 \mathrm{a} 1 \mathrm{~F}\left(5^{\prime}\right.$-CCTGGATGTTCTCTTCACTG-3') and 7a1R (5'GCCTGGATGCAGACTTTTCT- $3^{\prime}$ ). Quantitative PCR was conducted using SYBR Premix EX Taq (Takara Biotechnology, Dalian, China). The samples were analysed in triplicate, and their relative expression levels were normalised to the expression of $5 S r R N A$.

\section{Dual-luciferase reporter gene assay}

The luciferase reporter assay was performed at $48 \mathrm{~h}$ post-transfection. The cells were lysed using $1 \times$ reporter lysis buffer and were harvested by manual scraping. Luminescence was detected using a Mithras LB 940 (Berthold Technologies, Oak Ridge, TN, USA). The firefly luciferase activity of the pMIR-Report plasmid was measure 1 (M1), and the Renilla luciferase activity (internal control) of pRL-TK plasmid (Promega) was measure 2 (M2). The relative luciferase activity was calculated as the ratio of $\mathrm{M} 1 / \mathrm{M} 2$.

\section{Proliferation assay}

Cell proliferation was measured using the 3-(4,5-dimethylthiazol-2yl)-2,5- diphenyl-2H-tetrazolium bromide (MTT) method. At $48 \mathrm{~h}$ post-transfection, the transfection medium in each well was replaced by $100 \mu \mathrm{l}$ of fresh serum-free medium with $0.5 \mathrm{~g} \mathrm{l}^{-1}$ MTT. After incubation at $37{ }^{\circ} \mathrm{C}$ for $4 \mathrm{~h}$, the MTT medium was removed, and $50 \mu \mathrm{l}$ of DMSO was added to each well. After incubation at $37{ }^{\circ} \mathrm{C}$ for another $10 \mathrm{~min}$, the $A_{570 \mathrm{~nm}}$ of each sample was measured using a plate reader.

\section{Flow cytometry-based apoptosis and cell-cycle analysis}

At $48 \mathrm{~h}$ post-transfection, the $\mathrm{PC}-3$ cells were harvested and resuspended in phosphate-buffered saline (PBS) and then fixed in ethanol at $-20{ }^{\circ} \mathrm{C}$ overnight. The cells were washed with PBS and resuspended in staining solution $\left(50 \mu \mathrm{g} \mathrm{ml}^{-1}\right.$ propidium iodide, $1 \mathrm{mg} \mathrm{ml}^{-1}$ RNase A and $0.1 \%$ Triton X-100 in PBS). The stained cells $\left(1 \times 10^{5}\right)$ were then analysed for apoptosis and cell cycle with an FACScalibur; Becton Dickinson Flow Cytometer (PT. Madagasi Brosa Inc., Batang Hari, Propinsi Sumatera Utara, Indonesia).

\section{Nuclear staining with Hoechst 33342}

At $48 \mathrm{~h}$ post-transfection, apoptotic cells were detected using Hoechst 33258 staining (Beyotime, Shanghai, China) according to the manufacturer's protocols. Stained cells were imaged under a fluorescent microscope using 350-nm excitation and 460-nm emission. 
Statistical analysis

Statistical analysis was performed using $t$-test. Differences with $P<0.05$ were considered to be statistically significant.

\section{RESULTS}

The miRNA let-7a1 reduces IGF1R expression in PC-3 cells With performing analysis using TargetScan-Human 6.2 (http://www. targetscan.org/), IGF1R is one miRNA let-7a1 candidate target. PC-3 cells were transfected either with pSilencer4.1-let7a1 or parental vector, with either let-7al inhibitor or NC inhibitor, for $48 \mathrm{~h}$. The total RNA was extracted, and the whole-cell lysate was prepared. Western blot (Figure 1a) and real-time RT-PCR (Figure 1b) analyses were performed to detect the effects of miRNA let-7a1 or let-7a1 inhibitor on IGF1R expression. The results indicated that let-7al could reduce the protein level, while let-7al inhibition enhanced the IGF1R protein level; however, no apparent change in the IGF1R mRNA level was observed.

The miRNA let-7a1 directly interacts with its targeting sequences in the $3^{\prime}$ UTR of IGF1R mRNA

Three miRNA let-7al targeting sequences were found in the $3^{\prime}$ UTR region of IGF1R mRNA (Figure 2a) using TargetScan-Human 6.2. To confirm that miRNA let-7a1 directly targets these sequences, dualluciferase reporter assays were performed using cell transfection with the constructs in which these targeting sites or their mutants were cloned into the $3^{\prime}$ UTR of the luciferase reporter gene. The results revealed that the luciferase activities in the cells transfected with
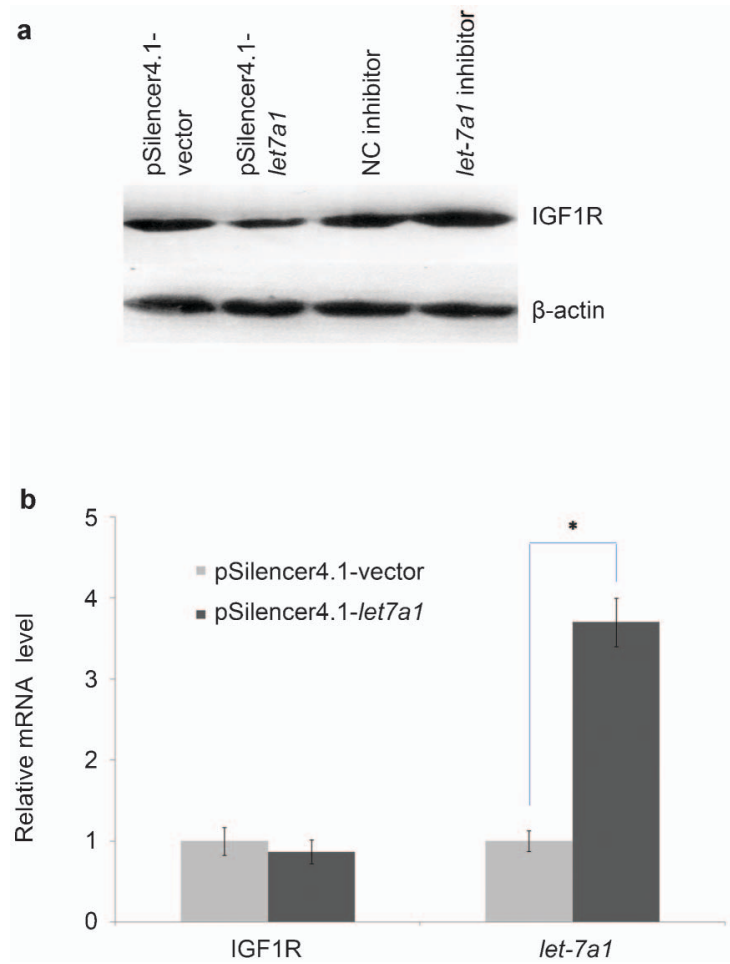

Figure 1 miRNA let-7al effects on IGFIR mRNA and protein expression. PC-3 cells were transfected with either pSilencer4.1-let7al or parental vector, with either let-7al inhibitor or NC inhibitor, for 48 h. Western blot (a) and real-time PCR (b) were conducted to detect the let-7al level and the effects of let-7al or let-7al inhibitor on the IGF1R expression. $\beta$-actin or $5 S$ rRNA expression was used as the internal control in the Western blots or in real-time PCRs, respec tively. The results shown in $\mathbf{b}$ are the means \pm s.d. $* P<0.05$. IGF1R, insulin-like growth factor-1 receptor; miRNA, microRNA; NC, normal control. a IGF1R mRNA (NM_000875)

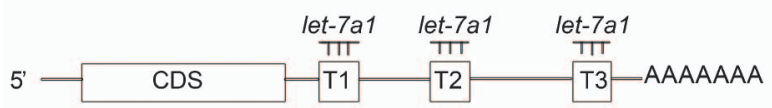

5 ...UUCACAAGCCUCCUGUACCUCAG... T1 (99-105 of IGF1R 3' UTR) 3' UUGAUAUGUUUGGAUG - -AUGGAGU miRNA let-7a1

5 '...CCCCCAAACAUUUAUCUACCUCA... T2 (2619-2626 of IGF1R 3' UTR)

3' UUGAUAUGUUGGAUGAUGGAGU miRnAlet-7a1

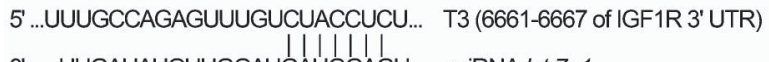

3' UUGAUAUGUUGgaUGAUGGaGU miRNAlet-7a1

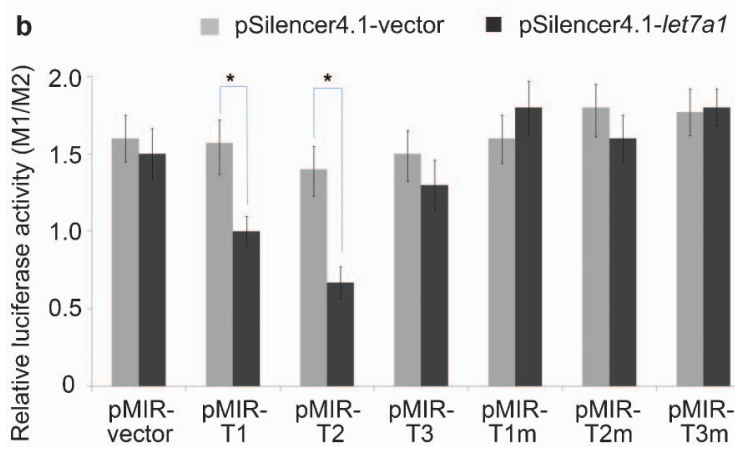

Figure 2 miRNA let-7al directly targets the 3' UTR of IGFIR mRNA. (a) Schematic representation of IGFIR mRNA showing the positions and sequences of the three predicted miRNA let-7al binding sites located in its 3' UTR. (b) PC-3 cells were co-transfected with pSilencer4.1-let7al (or parental vector), pMIR-T1 (or pMIR-T2 or pMIR-T3) or pMIR-T1m (or pMIR-T2m or pMIR-T3m) and pRL-TK for 48 h. Luciferase activity was detected using a Dual-Luciferase Assay System and was plotted as ratio of firefly to Renilla luciferase activity (M1/M2). The data are the means of three individual values \pm s.d. $* P<0.05$. IGF1R, insulin-like growth factor-1 receptor; miRNA, microRNA; $3^{\prime}$ UTR, $3^{\prime}$ untranslational region.

pMIR-T1 or pMIR-T2 were reduced as compared with the cells treated with the parental vectors, whereas transfection with pMIR-T3 or pMIR-T1m, pMIR-T2m and pMIR-T3m had no obvious effect on the luciferase activity (Figure $\mathbf{2 b}$ ). These results combined with the results in Figure 1 demonstrate that let-7a1 regulates IGF1R expression by directly targeting the T1 and T2 sites in $3^{\prime}$ UTR of the IGF1R mRNA to inhibit its protein expression.

The miRNA let-7a1 reduces Elk1 activity and $c$-fos expression Elk1 is a transcriptional factor activated by IGF1R signalling. Activated Elk1 binds with the Elk1 element to regulate the target gene expression. $\mathrm{C}$-fos is one of the Elk1-regulating genes involved in cancer cell phenotypes. To confirm that miRNA let-7a1 or let-7al inhibitor can influence Elk1 activity via changing the expression level of IGF1R, dual-luciferase reporter assays were performed using cell transfection with the construct in which the Elk1 element was cloned upstream of the TATA box of the pGL4-LUC2[minp] vector to form pGL4-Elk1. RT-PCR and Western blot were performed to detect the effects of let-7a1 or let-7a1 inhibitor on $c$-fos expression. The results revealed that let-7al could reduce, whereas the let-7al inhibitor increased, luciferase activity (Figure 3a), c-fos mRNA (Figure 3b) and protein (Figure 3c) expression.

\section{The effects of let-7a1 on PC-3 proliferation, cell cycle and cell} apoptosis

IGF1R is a tyrosine kinase receptor and mediates IGF1-induced signalling events in cell survival and proliferation. Our present results 
a

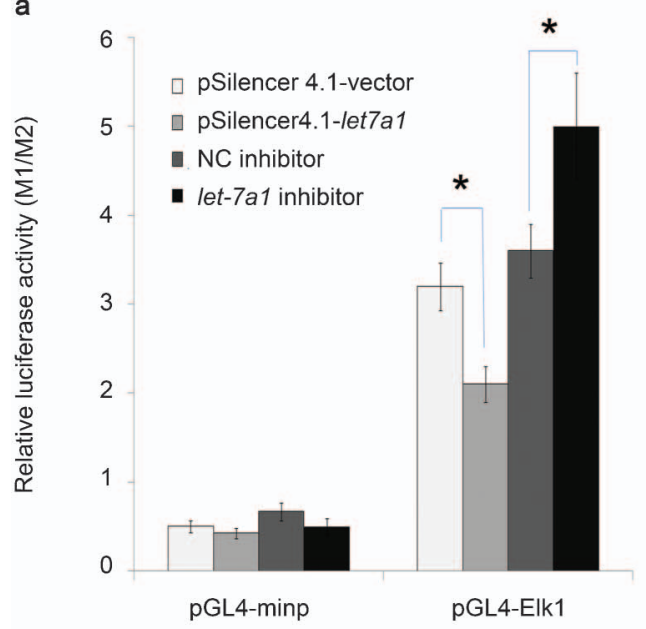

b

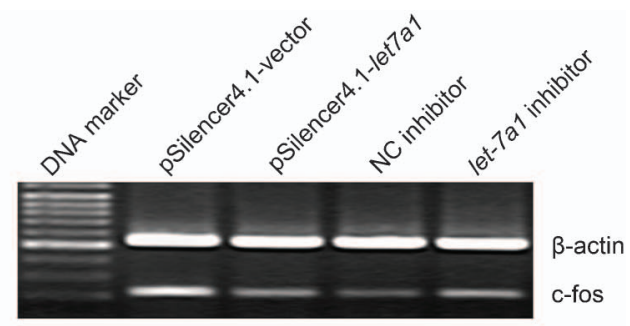

C

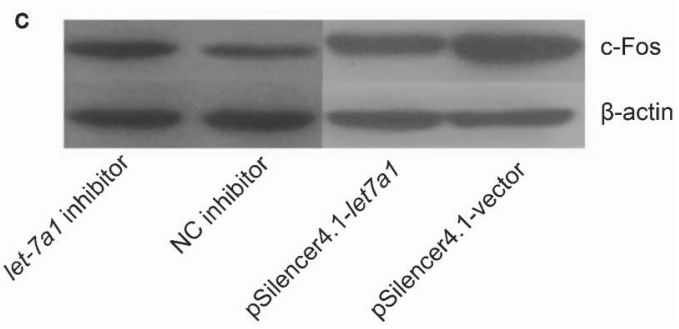

Figure 3 The effects of the miRNA let-7al on Elk1 activity and c-fos expression. Dual-luciferase reporter assays (a) were performed using cell transfection either with pSilencer4.1-let7al (or parental vector), or with let-7al inhibitor (or NC inhibitor), pGL4-Elk1 (or parental vector) and pRL-TK for $48 \mathrm{~h}$. The results are expressed as the relative luciferase activity (M1/M2). The data are represented as the mean of three individual values \pm s.d. $* P<0.05$. RT-PCR (b) and Western blot (c) were performed to detect the effects of miRNA let-7al or let-7al inhibitor on $c$-fos mRNA and protein expression. $\beta$-actin expression was used as the internal control in Western blot and RT-PCR. miRNA, microRNA; NC, normal control.

clearly demonstrate that let-7a1 reduces IGF1R expression in PC-3 cells by directly targeting the 3' UTR of IGF1R mRNA. We also detect the effects of let-7a1 or let-7a1 inhibitor on PC-3 proliferation, the cell cycle and cell apoptosis. As illustrated in Figure 4, the let-7a1mediated downregulation of IGF1R expression in PC-3 cells was accompanied by inhibition of cell proliferation (Figure $4 \mathbf{a}$ ), $\mathrm{G}_{0} / \mathrm{G}_{1}$ cell-cycle arrest (Figure $\mathbf{4 b}$ ) and enhanced cell apoptosis (Figure $4 c$ and d), whereas let-7a1 inhibitor resulted in $\mathrm{G}_{0} / \mathrm{G}_{1}$-cell decrease, S-cell increase and cell proliferation.

\section{DISCUSSION}

miRNAs are increasingly being discovered to be involved in regulating the malignant progression of cancer by directly targeting oncogenes and tumour suppressor genes. Aberrant expression of microRNAs has now been associated with many types of cancer. Some miRNAs exert oncogenic function by targeting tumour suppressor genes, and some exert anti-tumour function by targeting oncogenes. Let-7a has been reported to act as a tumour suppressor in some cancer types, such as lung and colon cancer, ${ }^{26-28}$ and the reduced expression levels of let-7 is correlated with poor clinical prognosis. ${ }^{29}$

Each miRNA can potentially interact with several mRNA targets via perfect or imperfect base pairing, primarily in the $3^{\prime}$ UTR portion. A number of target prediction algorithms relying on seed sequence pairing rules and conservational analysis have been developed to score possible recognition sites and identify putative gene targets. In the present study, we found that IGF1R is one of the let-7a1 target genes, and there are three potential target sites (T1, T2 and T3) of the miRNA let-7a1 in the IGF1R 3' UTR as predicted by TargetScan. To confirm that miRNA let-7al directly targets these sequences, dual-luciferase reporter assays were performed using cell transfection with constructs in which these targeting sites were cloned into the $3^{\prime}$ UTR of the reporter gene. Luciferase reporter assays, as well as real-time RTPCR and Western blot, demonstrated that let-7al regulates IGF1R expression by directly targeting the T1 and T2 sites in the 3' UTR of the IGF1R mRNA to inhibit its expression.
The inability of a cell to regulate its growth and proliferation is a distinctive feature of cancer. Activation of insulin-like growth factor-1 receptor (IGF1R) signalling is reportedly critical for prostate cancer cell growth and progression. IGF1R is a receptor tyrosine kinase that mediates IGF1-induced signalling events, including cell survival and proliferation. Our present results clearly demonstrate that let-7a1 downregulates IGF1R by directly targeting the $3^{\prime}$ UTR of IGF1R mRNA. This let-7a1-mediated IGF1R downregulation is accompanied by the attenuation of Elk1 activity and $c$-fos expression, inhibition of cell proliferation, enhanced apoptosis and cell-cycle arrest. This action is consistent with the well-established IGF1R/RAS/MAPK/ELK1 signalling pathway, which plays major roles in cell survival and proliferation.

Both let-7a and IGF1R are involved in prostate cancer and progression. Our finding that let-7a1 directly targets $I G F 1 R$ in prostate cancer cells could further reveal the mechanism of prostate carcinogenesis and progression. let-7a might partly contribute to IGF1R over-expression in prostate cancer cells, leading to the cell survival and proliferation. let-7a may be a novel therapeutic candidate for prostate cancer given its ability to induce cell-cycle arrest, inhibit cell growth and induce apoptosis, especially in hormone-refractory prostate cancer.

\section{AUTHOR CONTRIBUTIONS}

LNW assisted in the design of the study, conducted the cloning of reporter plasmid constructs, transfection studies, RT-PCR, MTT assays and Western blotting and helped draft the manuscript. WWC assisted in the design of the study, participated in the sequence alignment and use of computer database, assisted in the construction of recombinant plasmids and helped draft the manuscript. JZ performed flow cytometry, assisted with the transfection experiments, participated in the maintenance of cell lines and performed luciferase reporter assays. CYL conducted the isolation of RNA and purification of plasmids and assisted with the RT-PCR and Western blotting. CYL conducted the Western blotting and assisted with the purification of plasmids and luciferase reporter assays. JX assisted with the maintenance of the cell lines and the transfection experiments. PJZ participated in the construction of recombinant plasmids and the design of the study and 
a$$
\text { a }
$$
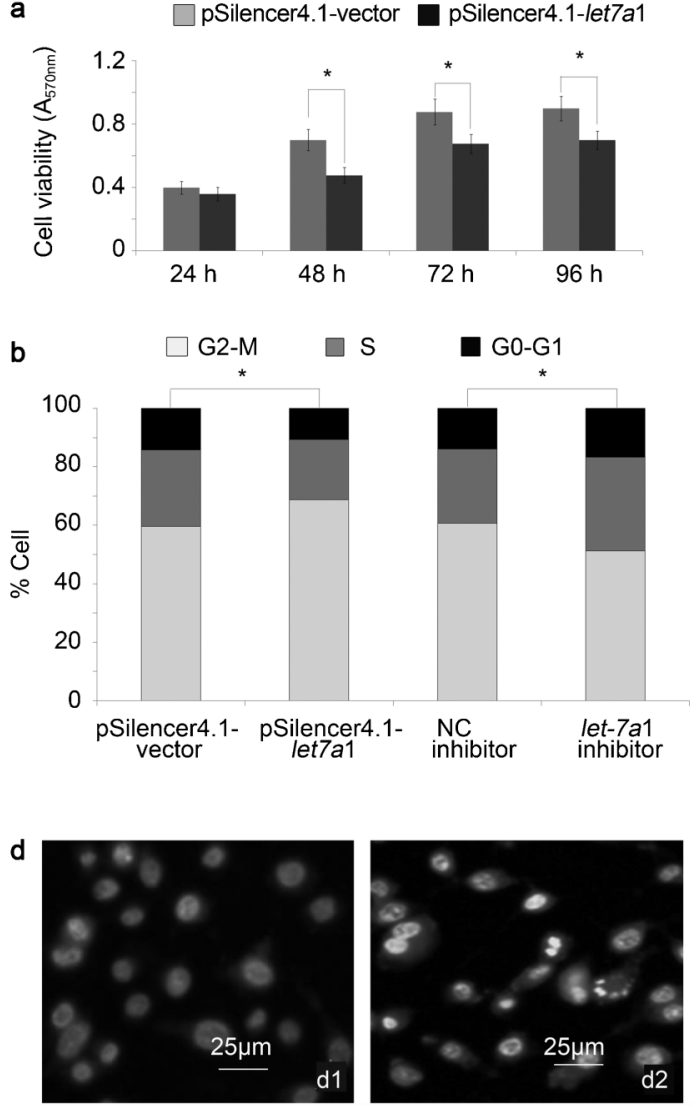

pSilencer4.1-vector

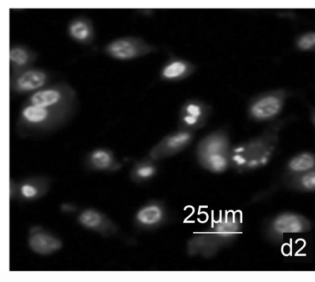

pSilencer4.1-let7a1

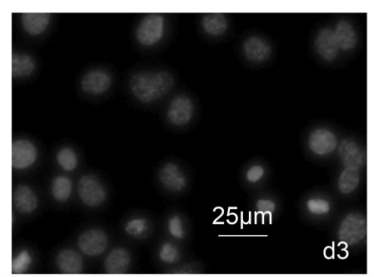

NC inhibitor

d3
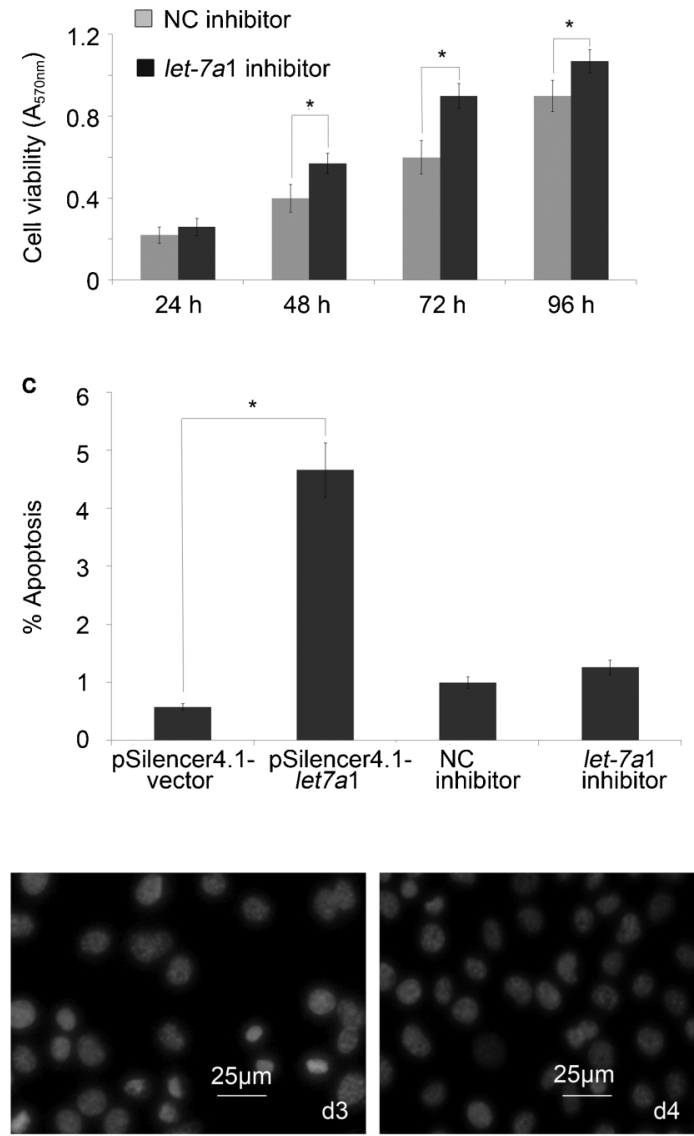

let-7a1 inhibitor

Figure 4 The effects of the miRNA let-7al on PC-3 proliferation, cell cycle and apoptosis. The changes in cell proliferation (a), cell-cycle distribution (b) and cell apoptosis (c, d) were examined as described in the experimental section in PC-3 cells that were transfected either with pSilencer4.1-let7al or parental vector with let$7 a 1$ inhibitor or NC inhibitor for $48 \mathrm{~h}$. All results are representative of three independent experiments. ${ }^{*} P<0.05$. miRNA, microRNA; NC, normal control.

assisted with the revising of the manuscript. ALJ participated in the design of the study and revision of the manuscript, helped draft the manuscript and assisted with the construction of recombinant vectors. All authors have read and approved the final manuscript.

\section{COMPETING FINANCIAL INTERESTS}

The authors declare that the research was conducted in the absence of any commercial or financial relationship that could be construed as a potential conflict of interest.

\section{ACKNOWLEDGMENTS}

This study was supported by grants from the National Natural Science Foundation of China (Nos. 81071720 and 81172045), Shandong Provincial Programs for Science and Technology Development (No. 2012GSF11820) and Foundation for Outstanding Young Scientist in Shandong Province (No. 2006BS03066).

1 Jemal A, Siegel R, Xu J, Ward E. Cancer statistics, 2010. CA Cancer J Clin 2010; 60 277-300.

2 Morgan C, Wagstaff J. Is there a role for ibandronate in the treatment of prostate cancer patients with bony metastases? Acta Oncol 2009; 48: 822-9.

3 Armstrong AJ, Carducci MA. Advanced prostate cancer: the future. Can J Urol 2005; 12: $42-7$

4 Zhu ML, Kyprianou N. Androgen receptor and growth factor signaling cross-talk in prostate cancer cells. Endocr Relat Cancer 2008; 15: 841-9.
5 Pollak M. Insulin-like growth factor-related signaling and cancer development. Recent Results Cancer Res 2007; 174: 49-53.

6 LeRoith D, Roberts CT Jr. The insulin-like growth factor system and cancer. Cancer Lett 2003; 195: 127-37.

7 Papatsoris AG, Karamouzis MV, Papavassiliou AG. Novel insights into the implication of the IGF-1 network in prostate cancer. Trends Mol Med 2005; 11: 52-5.

8 Ryan CJ, Haqq CM, Simko J, Nonaka DF, Chan JM et al. Expression of insulin-like growth factor-1 receptor in local and metastatic prostate cancer. Urol Oncol2007; 25 134-40.

9 Hellawell GO, Ferguson DJ, Brewster SF, Macaulay VM. Chemosensitisation of human prostate cancer using antisense agents targeting the type 1 insulin-like growth factor receptor. BJU Int 2003; 91: 271-7.

10 KrueckI SL, Sikes RA, Edlund NM, Bell RH, Hurtado-Coll A et al. Increased insulin like growth factor I receptor expression and signaling are components of androgen independent progression in a lineage-derived prostate cancer progression model. Cancer Res 2004; 64: 8620-9.

11 Nickerson T, Chang F, Lorimer D, Smeekens SP, Sawyers CL et al. In vivo progression of LAPC-9 and LNCaP prostate cancer models to androgen independence is associated with increased expression of insulin-like growth factor I (IGF-I) and IGF-I receptor (IGF-IR). Cancer Res 2001; 61: 6276-80.

12 Friedman RC, Farh KK, Burge CB, Bartel DP. Most mammalian mRNAs are conserved targets of microRNAs. Genome Res 2009; 19: 92-105.

$13 \mathrm{Ma} \mathrm{L}$, Teruya-Feldstein J, Weinberg RA. Tumour invasion and metastasis initiated by microRNA-10b in breast cancer. Nature 2007; 449: 682-8.

14 Martello G, Rosato A, Ferrari F, Manfrin A, Cordenonsi M et al. A microRNA targeting dicer for metastasis control. Cell 2010; 141: 1195-207.

15 Amankwah EK, Anegbe E, Park H, Pow-Sang J, Hakam A et al. miR-21, miR-221 and miR-222 expression and prostate cancer recurrence among obese and non-obese cases. Asian J Androl 2013; 15: 226-30.

16 Valastyan S, Reinhardt F, Benaich N, Calogrias D, Szász AM et al. A pleiotropically acting microRNA, miR-31, inhibits breast cancer metastasis. Cell 2009; 137: 1032-46.

17 Xiong Y, Fang JH, Yun JP, Yang J, Zhang Y et al. Effects of microRNA-29 on apoptosis, tumorigenicity, and prognosis of hepatocellular carcinoma. Hepatology 2010; 51 $836-45$. 
18 Park SM, Gaur AB, Lengyel E, Peter ME. The miR-200 family determines the epithelial phenotype of cancer cells by targeting the E-cadherin repressors ZEB1 and ZEB2. Genes Dev 2008; 22: 894-907.

19 Wang YY, Ren T, Cai YY, He XY. MicroRNA let-7ainhibits the proliferation and invasion of nonsmall cell lung cancer cell line 95D by regulating K-Ras and HMGA2 gene expression. Cancer Biother Radiopharm 2013; 28: 131-7.

20 Cai K, Wan Y, Sun G, Shi L, Bao X et al. Let-7a inhibits proliferation and induces apoptosis by targeting EZH2 in nasopharyngeal carcinoma cells. Oncol Rep 2012; 28: 2101-6.

21 Colamaio M, Calì G, Sarnataro D, Borbone E, Pallante P et al. Let-7a down-regulation plays a role in thyroid neoplasias of follicular histotype affecting cell adhesion and migration through its ability to target the FXYD5 (Dysadherin) gene. J Clin Endocrino Metab 2012; 97: E2168-78.

22 Kim SJ, Shin JY, Lee KD, Bae YK, Sung KW et al. MicroRNA let-7a suppresses breast cancer cell migration and invasion through downregulation of $\mathrm{C}-\mathrm{C}$ chemokine receptor type 7. Breast Cancer Res 2012; 14: R14.

23 Liu Y, Yin B, Zhang C, Zhou L, Fan J. has-let-7a functions as a tumor suppressor in renal cell carcinoma cell lines by targeting c-myc. Biochem Biophys Res Commun 2012; 417: 371-5.
24 Lu L, Katsaros D, Zhu Y, Hoffman A, Luca S et al. Let-7a regulation of insulinlike growth factors in breast cancer. Breast Cancer Res Treat 2011; 126: 68794.

25 Dong Q, Meng P, Wang T, Qin W, Qin W et al. MicroRNA let-7a inhibits proliferation of human prostate cancer cells in vitro and in vivo by targeting E2F2 and CCND2. PLoS ONE 2010; 5: e10147.

26 Inamura K, Togashi $\mathrm{Y}$, Nomura K, Ninomiya $\mathrm{H}$, Hiramatsu $\mathrm{M}$ et al. let-7 microRNA expression is reduced in bronchioloalveolar carcinoma, a non-invasive carcinoma, and is not correlated with prognosis. Lung Cancer 2007; 58: 392-6.

27 Esquela-Kerscher A, Trang P, Wiggins JF, Patrawala L, Cheng A et al. The let-7 microRNA reduces tumor growth in mouse models of lung cancer. Cell Cycle 2008; 7: 759-64.

28 Yang N, Kaur S, Volinia S, Greshock J, Lassus H et al. MicroRNA microarray identifies Let-7i as a novel biomarker and therapeutic target in human epithelial ovarian cancer. Cancer Res 2008; 68: 10307-14.

29 Takamizawa J, Konishi H, Yanagisawa K, Tomida S, Osada H et al. Reduced expression of the let-7 microRNAs in human lung cancers in association with shortened postoperative survival. Cancer Res 2004; 64: 3753-6. 